\title{
Exploration of Hainan Translation Service Talent Cultivation and the Demand Based on International Tourism Island Construction
}

\author{
Qingping Zhang \\ Hainan Foreign Language College of Professional Education, Wenchang, 571321, China
}

Keywords: international traveling island construction; Hainan translation service industry; talent; cultivation and demand

\begin{abstract}
Hainan island is rich in tourism resources. It is the representative of typical tropical coastal city. The proposal of national tourism island strategy brings development opportunity for Hainan's economy. The opportunity and challenge coexist. How to plan Hainan translation service talent training and demand strategy becomes a hot issue. Several opinions on driving construction of Hainan International Tourism Island issued by the State Council require Hainan to become world-class tourist attraction within 15-20 years. Thus, this paper based on the general background of international tourism island construction proposes related suggestions in allusion to translation service talent cultivation through analyzing talent cultivation and demand of translation service industry.
\end{abstract}

\section{Introduction}

Through development for many years, Hainan gradually achieves expected goal of international tourism island construction, and tourism GDP continues to rise. Now, development momentum of Hainan tourism service industry is rapid. Annual number of domestic and overseas tourists increased to 40601800 person-times in 2014 from 750800 person-times in 1987, and total tourism income rose to 50.653 billion Yuan from 114 billion Yuan. Over the 20 years, the total number of tourists and total tourism income increased by 54.08 times and 444.32 times respectively. Top 8 tourist sources of Hainan include: Korea, Japan, Singapore, Russia, Malaysia, Britain, Thailand and Germany. Now, international tourists occupy an important part among Hainan tourists. In the face of these foreign tourists, foreign language service becomes inevitable. However, numerous problems exist in translation service talent cultivation and demand based on the background of Hainan International Tourism Island construction. Thus, it is very significant to deeply investigate them.

\section{Demand of international tourism island construction for Hainan translation service talents}

\section{Rise in quantity demand}

As international tourism island construction further advances, Hainan tourism gains rapid development. Hence, the demand for Hainan translation service talents is also on the rise. Data statistics shows by 2020, data statistics of talents in Hainan will reach 1.85 million. If athletic contest and gaming industry are included, total demand of tourism talents and tourism talents will reach 419000. Therefore, Hainan International Tourism Island construction badly needs a large number of service talents and especially translation service talents.

\section{Rise in demand types}

Hainan tourism will inevitably develop towards diversification and standardization. Through careful analysis of human resource distribution of tourism, it is known that talent demand mainly concentrates on the following: 1) high-end management talents. Operation and management gap of Hainan tourism makes system theory and professional ability of management personnel very inharmonious. The shortage of high-end management talents makes bottleneck occur in Hainan International Tourism Island construction; 2) development and planning talents. With further implementation of national policy, international tourism island becomes a hot construction project in Hainan. Tax-free business in Sanya and other regions further promote development and growth of 
tourism market. Tourist' consumption and view tend to be rational. The measure of enclosing land and building houses is not suitable for mass consumption. Scientific and reasonable blueprint planning becomes the key emphasis in work. Tourist' enjoyment of the beauty of nature and development of rural independent tourism becomes the mainstream direction; 3) high-quality skill service talents. The development key of Hainan tourism lies in prosperity of service industry. This is also the key point of Hainan International Tourism Island construction. At present, the quality of employees in Hainan service industry is uneven. This increases enterprise cultivation input and results in operation risks. Therefore, it is required to pay attention to cultivating high-quality skill service talents and strive to improve their stability; 4) other service talents. International tourism island mainly aims at international tourists. The talents familiar with entry and exit business, international tourism and tour guides of some minority languages are also needed. Management talents and translation talents who can carry out information exchange on large-scale commercial activities are also needed. Talent cultivation quality of colleges becomes the development key.

\section{Cultivation demand of international tourism island construction for Hainan translation service talents}

\section{Imbalanced cultivation structure results in shortage of talents}

As construction speed of Hainan International Tourism Island speeds up, more international tourists go to Hainan for leisure tourism. Thus, Hainan service talent cultivation shortage phenomenon becomes increasingly serious. From 2010, Hainan translation service industry started to introduce domestic and foreign translation talents in quantity. Data statistics shows in the past four years, Hainan has introduced 86200 professional translation talents in total. Superficially, this figure is very large, but compared with huge translation marked demand of Hainan, the gap is very obvious. Among translation talents introduced, some talents have no high professional translation quality, so they cannot carry out high-end translation work. Most of them work on low-end translation such as tourism promotion. English translation talents are relatively numerous, but minority language translation talents are badly short. Take an event reported on network in 2013 for example. A person from the Syrian Arab Republic went to Hainan for trade activity; he sought several Arabic interpreters, but all of them could not be qualified for the job. Finally, the agreement failed to be signed for a great international trade project on schedule. This gave rise to great loss for both companies. Some translation talents fail to properly understand related professional terms and local slangs, so they cannot offer accurate translation services for international tourists. Thus, some international tourists feel disappointed. For instance, during translating some scenic spots in a famous tourist attraction "Wuzhi mountain" in Hainan, since some scenic spots are named with local slangs, related translators cannot fully comprehend these slangs. Thus, irrelevant answers often occur in translation. On the whole, Hainan translation service industry is faced with talent shortage in development. In particular, high-quality professional translation talents are badly short.

\section{Vicious competition among enterprises leads to unclear training objective}

Hainan translation service industry presents unprecedented development vigor. Various kinds of professional translation talents play increasingly great roles in constructing Hainan International Tourism Island. During 2015 Boao Forum for Asia, since a large number of high-quality translation talents participated, the forum could be conducted smoothly. The survey data show on annual meeting of Boao Forum for Asia, during receiving foreign guests, text translation and tourism promotion, the number of related translation personnel exceeded 12000. An increasing number of foreign friends enjoy meticulous kindness in Hainan. However, Hainan translation industry fails to form scale operation, and vicious competitions of many translation companies are unceasing. Many translation companies enhance welfare and treatment of talent recruitment in order to strive for more excellent translation personnel. Some translation companies even propose million Yuan annual salary to attract talents. This makes many translation talents conduct job-hopping. Some even change 7-8 units within one year. Take a famous translation company in Hainan - YGYM Sanya Translation Company for example. In 2014, translation talent turnover rate of the company was as high as $25.47 \%$. 
The situations of other small translation companies were even worse. Many local tourism companies also strive for translation talents in order to strive for local limited tourism resources. This makes development of Hainan service industry development disordered. In general, competitions in Hainan translation service industry are very fierce. The demand for various translation talents and especially minority language translation talents exceeds the supply. Vicious competitions of enterprises make colleges have no explicit cultivation goal of translation service industry and disconnect with the market.

\section{Measures to enhance Hainan translation service talent cultivation}

\section{Coordinate talent cultivation mechanism based on market background}

Related data of Hainan show enterprises in Hainan have high requirements for foreign language application talents. Solid foreign language foundation, business knowledge and business etiquette become rigid indexes of Hainan translation service talent demand. Thus, practical ability, operational ability and communication ability become main direction of talent mechanism adjustment under Hainan market background. Hainan translation service talents must own the abilities to independently handle foreign letters, complete preparation of expected business activities and business sales. Seeing from post demand, the foreign is extensive, and the plasticity is strong. The demand for related posts of Hainan translation service talents is also huge. This offers favorable development space for those of foreign language specialty in Hainan. For instance, Hainan College of Foreign Studies where the author works practically cultivates talents connected with the market on the basis of Hainan international trade and service industry, including inter-disciplinary talents with solid foreign language foundation, skillful business operation ability, skillful business handling ability and strong communication ability. Translation knowledge + business etiquette + operating skills are prominent features of prominent features, and great achievements have been gained.

\section{Set up science course mechanism based on employment background}

Course mechanism is an important guarantee of talent quality. Combining theory and practice is a principle of cultivation mechanism of Hainan translation talents. It is required to attach importance to more comprehensive course setting, make connection of various disciplines stronger, expand core course teaching, regard foreign language listening, speaking and translation courses as key teaching points and make optional course module more diversified. It is necessary to synthesize specific conditions of Hainan International Tourism Island construction, bring hotel marketing and tourism business into optimal courses, actively introduce foreign advanced school-running modes and excellent courses, master dynamics of national policy and properly construct scientific course mechanism of talent cultivation for Hainan International Tourism Island construction. Professional foundation and quality, vocational ability and core courses are four directions of module construction system. The whole course system takes cultivation of professional consciousness of Hainan translation service talents as the objective. Ability + knowledge inter-disciplinary talent standards are established gradually. Professional certificate and academic education are fully combined. Professional adaptability of Hainan translation service talents is more diversified.

\section{Adopt work-study combination mechanism based on development status of Hainan economy}

Talent cultivating mechanism construction for Hainan translation service industry needs to actively adapt society and local economic development status. In addition, it is necessary to conduct innovation of talent cultivation mode on the basis of industrial structure change and finally achieve inter-disciplinary skills of Hainan translation service talents. Work-study combination mechanism oriented to employment post becomes the mainstream. It is required to actively encourage students to take part in local activities, serve for business exchange or competitions, facilitate development of local economy and improve self-translation skills. At the same time, industrial association and relevant cooperative enterprises also need to communicate with colleges in time in development process, change and develop teaching plan in time. Hainan translation service talents coordinate with economy and market in order to drive development of Hainan tourism fundamentally. 


\section{Introduce first-class training strategy based on current situation of diversified translation talents}

To drive development of Hainan translation service industry, it is required to first solve translation talent shortage problem. The author suggests Hainan should formulate more positive diversified translation talent introduction and cultivation strategy. In the aspect of translation talent introduction, Hainan should negotiate with domestic and overseas famous colleges and let excellent translation graduates of these colleges come to Hainan and join Hainan translation service industry development. Some translation companies with powerful strength can run a school jointly with colleges, attract more high-quality translation talents and ensure relative stability of talents. Seeing from self-training of translation talents, translation talents must be done well in order to cultivate a large number of high-quality translation talents as soon as possible. Translation level can improve only through continuous training so as to be better qualified for translation. Especially for high-end business translation, it is necessary to carry out special training for related translation personnel so as to let them master standard and authentic professional term translation as soon as possible.

\section{Set up translation service center based on development of international tourism island}

Government sector may set up translation service center. Related departments should pay high attention to the important functions of translation service industry on economic development, further enhance translation service work construction, construct Hainan translation service center through setting up official project regulations, integrate existing talents ad education, and enhance interactive cooperation of education institutions, translation institutions and scientific research institutions of Hainan and other provinces so as to drive rapid development of Hainan translation service industry. At present, school running institutions of Hainan involve more than 10 foreign language teaching, including English, Japanese, Russian and French etc. However, seeing from future development, Hainan will receive tourists from all over the world, so only 10 languages are far from enough. It is very important to expand translation service range and extend translation service language varieties. Moreover, existing educational resources and translation platform may be depended on to further develop translation service industry, further promote rapid and healthy development of translation service industry, strengthen attention to translation service work based on existing educational resources and translation platform, pay attention to talent cultivation and prepare to build translation service center as soon as possible.

\section{Conclusions}

To sum up, Hainan translation service talent cultivation on the basis of international tourism island should be based on wide knowledge scope, strong cross-cultural communication and rich practice and employment competition, cultivate inter-disciplinary talents with innovation spirit and sustainable development, and provide convenience for social and economic development of Hainan under joint efforts of related employees so as to jointly construct the blueprint of international tourism island.

\section{Acknowledgments}

This paper is one of the research results produced from the 2015 Planned Research Project of Hainan Provincial Philosophy and Social Sciences Titled as The Countermeasure Research on Development of Hainan Translation \& Interpretation Services Based on Construction of International Tourism Island, Project No.: HNSK(Z)12-53.

\section{References}

[1] Cao Shenyan, Construction of business English talent cultivation mode based on needs of regional economic development. Education and Vocation, 2011(06):104-105. 
[2] Liang Guang, Thought on professional building and development under background of Hainan International Tourism Island construction - case study of Hainan Normal University. Journal of Hainan Normal University (social sciences), 2011(03):170-172.

[3] Xie Luping, Relationship between cultural activity of college students and Hainan International Tourism Island construction - case study of college culture construction of Hainan Normal University. Journal of Hainan Radio and TV University, 2011(02):103-105.

[4] Xie Luping, On relationship between students' cultural activities in colleges and Hainan International Tourism Island construction - case study of cultural construction of College of Chinese and Literature, Hainan Normal University. Manager' Journal, 2013(26):254.

[5] Wang Xiumin, Survey of cross-cultural communication ability of Hainan English tour guides. Science \& Technology Vision, 2013 (06): 98-99.

[6] Huang Jianhong, Gong Ping, Analysis of demand of countermeasures Hainan International Tourism Island construction for tourism talents and the countermeasures. Education Science \& Culture Magazine (middle-ten-day periodical), 2009(05): 35, 59.

[7] Wu Jue, Wang Fengxia, Education system construction of Hainan tourism specialty under background of international tourism island. Great Masters, 2011 (09): 123. 